\title{
Outdoor Parking Payment with IoT in Malaysia
}

\author{
Ong Chun Heng ${ }^{1, *}$ Intan Farahana Binti Kamsin ${ }^{2}$
}

\author{
${ }^{1,2}$ Asia Pacific University of Technology and Innovation, Technology Park Malaysia, Bukit Jalil, Kuala Lumpur, \\ Malaysia. \\ ${ }^{*}$ Corresponding author. Email:tp050832@mail.apu.edu.my
}

\begin{abstract}
Internet of Things (IoT) will bring a paradigm shift in technology that will potentially change the behaviour of things in our daily lives. It has been shown by current technology like smart devices, are starting to adopt sensors to enhance the experience of the users. This research aims to improve the efficiency of the process of paying for outdoor parking and reduce the effort of paying manually at ticketing booth. This research will also discuss samples collected based on college students and working adults and their opinions of the system. It is to evaluate the technology exposure and knowledge of the user in order to be comfortable with using the system. This research will be contributing to the development of smart city by integrating IoT technology into parking system in order to improve the experience and future research is needed to enhance the security of the system.
\end{abstract}

Keywords: Internet of Things, NFC, Parking, Payment, Sensor.

\section{INTRODUCTION}

Parking payment system behaviour has been influenced by the Information Technology on how it operates and provide services to its user. With the digital payment being on the popular trend, there is no doubt that every existing system will be digitalized. [1] However, the people in Malaysia are one of the populations that are still paying with parking ticket with self-service machine. [2] When system is fully digitalized, it will be easier to connect things together with the emerging IoT technology. The solution proposed in this research does not modify the parking behaviour of users, instead it will enhance the experience of the overall process of payment for parking. Thus, proposed system will be utilizing the IoT technology in order to improve current parking payment system [16-21]

\section{RELATED WORKS}

This section reviews past researches in order to come up with this topic. Several domains are identified and these domains are Internet of Things, Internet of Things in Malaysia, Outdoor Parking, Payment and Outdoor parking payment.

\subsection{Internet of Things}

The unique thing about IoT is that it is made with lowpower concept in mind so that the design of the devices can be small, reliable and low cost [3]. A notable example of achieving the low-power usage is by using the MQTT protocol which is a messaging protocol for low bandwidth environments. According to [4], experiment of size payload to energy consumption has been conducted using MQTT protocol which shows that it consumes less energy as the payload sizes increase. References [5] had recently made a breakthrough on enabling IoT systems to operate without batteries that instead harvest energy through other sources such as radio waves, light sources and vibrations. When lowpower devices become popular, society will be ready to embrace the IoT technology.

\subsection{Internet of Things in Malaysia}

Smart meters have been implemented as an alternative to traditional electric meters which uses IoT technology helps consumers to monitor and regulate energy consumption remotely [6] [24-28]. According to [6] study, one of the factors that influence consumer's acceptance to smart meter is greatly increased when consumers are educated with environmental awareness and electricity-saving knowledge. Another recent IoT technology RFID tag [22, 23] linked with E-wallet 
account is also utilized for paying e-toll on toll plaza where the sensor can pick up signal from the RFID tag placed on windscreen or headlamp of the vehicle [7]. From the study by [7], it is discovered that RFID usage on tolling and parking is preferred by users as high as 93\% and $91 \%$ compared to RFID usage on electronic road parking where only $45 \%$ of users show willingness.

\subsection{Outdoor Parking}

References [8] stated that vehicle population is increasing over the years thus the increase of demand of space for parking. From the study, it is discovered that development of multi-level parking provides ample space for drivers. However, people are having tendency to park as close to the destination as possible which encourages on-street parking either legally or illegally. References [9] stated that curb parking in cities is hard to find due to several reasons such as required to pay at meters, confusing regulation like permits required, fees charged at weekday but not weekend and so on.

\subsection{Payment}

Payment has also come a long way where dealings no longer need to be dealt with physical cash anymore. References [10] stated that payment has been revolutionized and have different methods of payment such as online mobile banking, mobile payment using QR pay or NFC payment, and mobile money which is linked to a mobile phone number. References [10] mentioned 4 factors, perceived usefulness, perceived ease of use, performance expectancy and social influence which influence people to lean towards to digital currency as it gives them the flexibility in payment methods.

\subsection{Outdoor Parking Payment}

References [11] and References [12] stated that method of payment for parking is still utilizing parking tickets. Parking payment machine is still used in current parking system in order to validate their own parking. References [13] found that pricing policy heavily influenced the number of vehicles at on-street parking. Nanning, an urban city in China has been using on-street parking system but struggle to accommodate increasing parking demand. It is overcome by the adjustment of price policy increase, where people with high income are not affected by the price policy change while the lowincome people does not have the tendency to park which leads to the decreasing of the vehicles at on-street parking so that overall, the people with high income has higher satisfaction.

\subsection{Similar System}

This section will discuss the similar systems which focus on various design of parking system and parking payment. $[11,14,15]$

\subsubsection{System 1}

This system consists of 3 hardware components, Raspberry Pi camera, ultrasonic sensor and GPS module which are connected to the Raspberry Pi. ultrasonic sensor is used for detecting parking slot, Raspberry Pi camera is used to support the detection of the sensor by identifying the presence of vehicle and GPS module is to locate the current location of the parking slot. When parking slot is empty, LED indicator will be off and send status update to Blynk application and location of the parking slot.

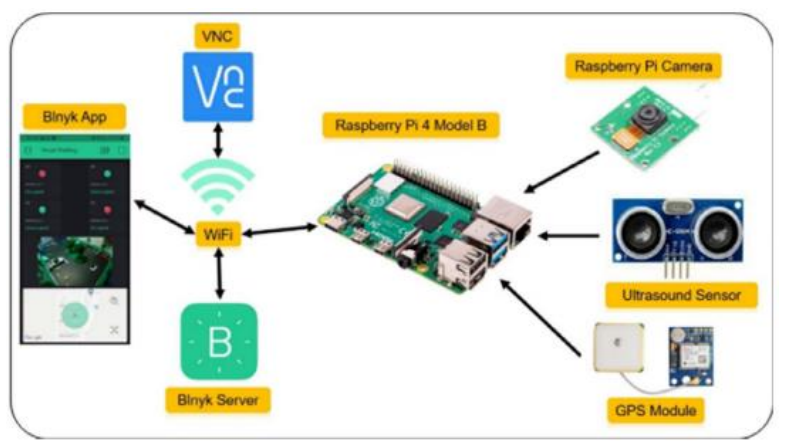

Figure 1 Raspberry Pi Based Smart Parking Management System Architecture

\subsubsection{System 2}

In this car park system, entry and exit is checked using RFID tag to prevent vehicle from re-entering the car park. The Android application is connected to cloud via Firebase database. When a booking is made, the information is stored and updated in real time to Firebase and payment is made online. Upon arriving the spot, the Raspberry Pi (RPi) will retrieve the information from firebase to verify the vehicle and allow for passing through the gate. IR and ultrasonic sensors are placed at parking slot to check the presence of vehicle. IR sensor is also used to check the height of vehicle to prevent it from entering car park if it reaches above a certain set value. Cost is calculated based on the stored database information of entry time and exit time. 


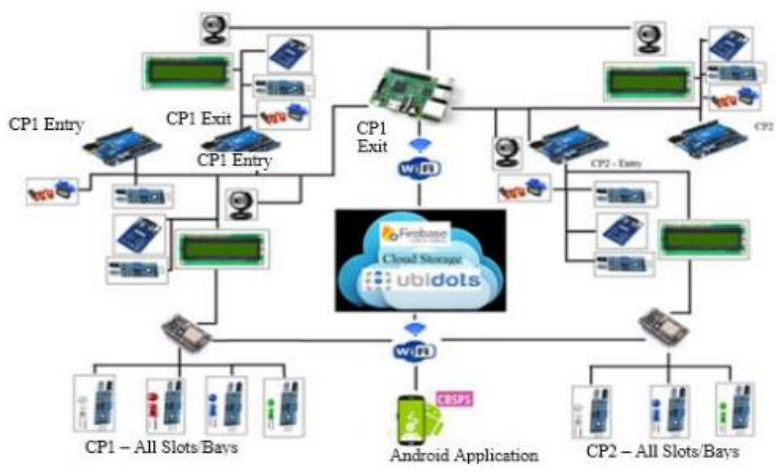

Figure 2 Cloud-Based Smart Parking System

\subsubsection{System 3}

$\mathrm{RPi}$ is implemented to manage the data input and output from the cloud database, Firebase. Upon entering, users need to scan QR code to access the barrier and timer will be logged. Parking spot is automatically provided and user can choose to accept or reject the parking slot. Ultrasonic sensors are used to detect and change the LED light based on the presence of vehicles. The status of parking spot is updated to database in real-time whenever the LED light changes. Fees are calculated based on timer and payment is made through mobile application.

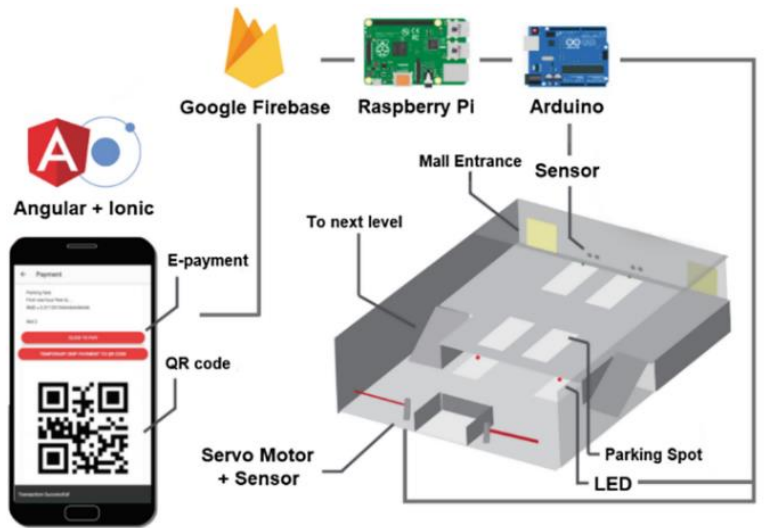

Figure 3 Architecture of the proposed system

\subsubsection{Comparison of Similar Systems}

Table 1 Comparison between Similar Systems

\begin{tabular}{|l|l|l|l|}
\hline Criteria/System & $\begin{array}{l}\text { System 1 } \\
\text { (S1) }\end{array}$ & $\begin{array}{l}\text { System 2 } \\
\text { (S2) }\end{array}$ & $\begin{array}{l}\text { System 3 } \\
\text { (S3) }\end{array}$ \\
\hline IoT Sensors & $/$ & $/$ & $/$ \\
\hline Notification & & & \\
\hline Database/Server & $/$ & $/$ & $/$ \\
\hline $\begin{array}{l}\text { Real-time } \\
\text { information }\end{array}$ & $/$ & $/$ & \\
\hline Payment & & $/$ & $/$ \\
\hline
\end{tabular}

Based on Table 1, it shows that S2 has most of the criteria like IoT sensors, database, real-time information and payment while S1 does not have payment and S3 does not have real-time information. Thus, the proposed system will integrate all 5 features like IoT sensors, notification, server database, real-time information, and also include an automation payment method.

\subsection{Overview of the Proposed System}

In order to use NFC, it must be closed range and attach on a unique object that does not require to be change often. In the case of vehicle, the object will be the car plate. It is also in a perfect position to be read by a reader. It is also noted that NFC plate must withstand washing and water resistant since vehicles are under outdoor environment. In each of the parking spot, it is fitted with a reader that is able to detect and read NFC devices. These readers are connected to a server to keep log information such as time, car plate info and fees charged. It can be stored on cloud in order to backup that $\log$ information. When a car is approaching the parking spot, it will not charge immediately. The NFC device and NFC-enabled reader will constantly log the information and keep track of the time. Users are also able to track the status of time through their mobile phone. After a certain period of time, the server will send notification to the user to indicate the number of hours parked and the accumulated fees charged. Since it is in an outdoor parking, the following rules of payment must be listed, under 15 minutes no fee charges will apply while more than 30 minutes' fee charges apply. In order to address the issue where the NFC cannot be read properly, there will be sensor indicator to show the user if such problem arises. (LED light) On the user-end, user does not have to deal with machines anymore and only interact using mobile client. After the vehicle is parked, there will be an app in mobile phone that receive the logged information from the server based on their car plate. The charge can also be accumulated and paid later at the end of the month.

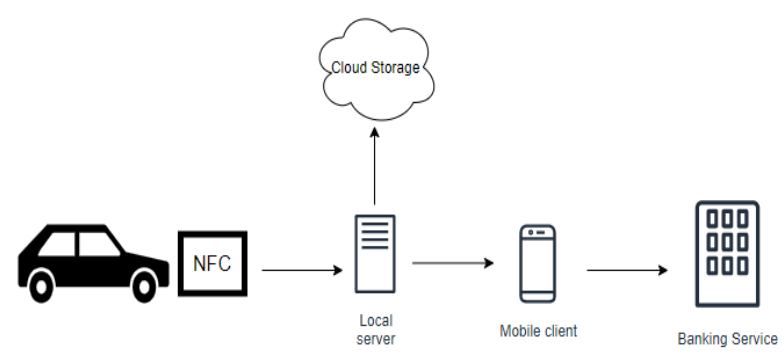

Figure 4 Process of payment 


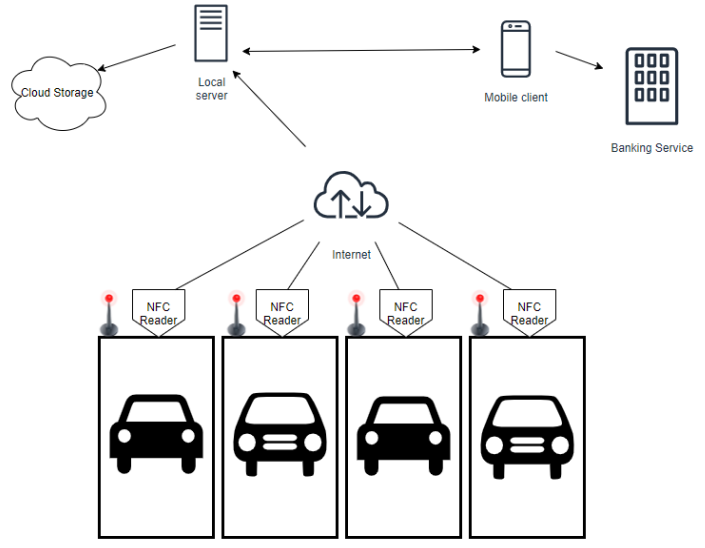

Figure 5 Overview of the system

\section{METHOD}

Judgment sampling is used by carefully choosing the respondent that are eligible for the research sample. The targeted sample in this research will be college students in Malaysia and the sample size taken will be 8 people. The chosen respondents must currently pursue in the field of IT. This is because they are mostly tech independent and the sample will be able to provide reliable data.

\section{DISCUSSION}

Parking meters are becoming obsolete in the public as it is time consuming and difficult for the people to use. Thus, automated payment will bring a huge impact to the design of IoT smart city. The solution proposed will improve the payment process to become more efficient for the people in the outdoor parking environment and reduce the hassle of dealing with machines by using the NFC technology for payment.

\section{CONCLUSION}

It has been discussed integration with IoT technology might enrich the experience depending on the knowledge of the user. Users in these days are heavily dependent on smart services such as e-payment, real-time tracking, intelligent personal assistant and so on. Therefore, existing physical robust systems should be replaced and users need to be educated in order to catch up with the digital trend in the society. The change in technology and methods will definitely advance our country further into the vision of smart city. The building of smart city does not happen overnight; small steps are taken to change the city infrastructure. The current limitation is that sensor that are placed in the public may be damaged by weather such as rain thus it will require constant maintenance by the relevant personnel to ensure it is working in good condition. Future works and research should be focusing on how to enhance the system with a better security protection against NFC eavesdropping.

\section{ACKNOWLEDGMENTS}

Highest appreciation to Asia Pacific University, Malaysia for the opportunity on the article publication.

\section{REFERENCES}

[1] Tiewul, M., Factors Influencing Digital Marketing and Digital Payment on Consumer Purchase Behaviour. International Journal of Applied Research in Management and Economics, 3(3), 2020, $39-45$.

DOI:

https://doi.org/10.33422/ijarme.v3i3.501

[2] Igor, Stevan, Gordana, Sabolc, Support for Selfservice Automated Parking Systems, 19th International Symposium INFOTEH-JAHORINA (INFOTEH), 2020, pp. 1-5, DOI: https://doi.org/ 10.1109/INFOTEH48170.2020.9066319

[3] Nasab, M.A., Shamshirband, S., Chronopoulos, A.T., Mosavi, A. \& Nabipour, N, Energy-Efficient Method for Wireless Sensor Networks Low-Power Radio Operation in Internet of Things. Electronics, Vol. 9, 2020, p.p. 320. DOI: https://doi.org/ 10.3390/ELECTRONICS9020320

[4] Pegah Nikbakht Bideh, Jonathan Sönnerup, and Martin Hell, Energy consumption for securing lightweight IoT protocols, In Proceedings of the 10th International Conference on the Internet of Things (IoT '20). Association for Computing Machinery, New York, NY, USA, Article 16, 2020, 1-8. DOI: https://doiorg.ezproxy.apiit.edu.my/10.1145/3410992.341100 8

[5] SheinEsther, A battery-free internet of things. Communications of the ACM. 64 (7), 2021, p.pp 16-18, DOI: https://dl-acmorg.ezproxy.apiit.edu.my/doi/abs/10.1145/3464937

[6] Alkawsi, G.A., Ali, N. \& Baashar, Y, An Empirical Study of the Acceptance of IoT-Based Smart Meter in Malaysia: The Effect of Electricity-Saving Knowledge and Environmental Awareness, IEEE Access, 8, 2020, p.pp. 42794-42804. DOI: https://doi.org/ 10.1109/ACCESS.2020.2977060

[7] Waqas Ahmed, Sheikh Muhamad Hizam, Ilham Sentosa, Habiba Akter, Eiad Yafi and Jawad Ali, Predicting IoT Service Adoption towards Smart Mobility in Malaysia: SEM-Neural Hybrid Pilot Study, International Journal of Advanced Computer Science and Applications (IJACSA), 2020, 11(1). 
DOI:

http://dx.doi.org/10.14569/IJACSA.2020.0110165

[8] Parmar, J., Das, P. \& Dave, S.M., Study on demand and characteristics of parking system in urban areas: A review. Journal of Traffic and Transportation Engineering (English Edition). 7 (1), 2020, p.pp. 111-124. DOI: https://doi.org/ 10.1016/J.JTTE.2019.09.003

[9] Michael Manville, Miriam Pinski, The causes and consequences of curb parking management, Transportation Research Part A: Policy and Pratice, 2021, ISSN 0965-8564, DOI: https://doi.org/10.1016/j.tra.2021.07.007

[10] Reiting, P., Mladenow, A., Strauss, C. \& Kotsis, G, Mobile payment: Classic approaches to promote consumer adoption. ACM International Conference Proceeding Series, 2020, p.pp. 84-93. DOI: https://doi.org/10.1145/3428690.3429182.

[11] Yu, J.L., Ng, K.H., Liong, Y.L. \& Hanafi, E, IoT Based Cloud-Integrated Smart Parking with ePayment Service. Advances in Intelligent Systems and Computing, 1229 AISC, 2020, p.pp. 405-414. DOI: https://doi.org/ 10.1007/978-3-030-522469_30

[12] M A M B Kamaruzaman and Naziffa Raha Md Nasir, PARKEY: ticket-less parking system using license plate recognition approach, J. Phys.: Conf. Ser, 2021, 1860, $012006 . \quad$ DOI: https://doi.org/10.1088/1742-6596/1860/1/012006

[13] Mo, B., Kong, H., Wang, H., Wang, X. (Cara) \& Li, $\mathrm{R}$, Impact of pricing policy change on on-street parking demand and user satisfaction: A case study in Nanning, China, Transportation Research Part A: Policy and Practice. 148., 2021, p.pp. 445-469. DOI: https://doi.org/10.1109/2.161279

[14] Waheb A. Jabbar, Chong Wen Wei, Nur Atiqah Ainaa M. Azmi, Nur Aiman Haironnazli, An IoT Raspberry Pi-based parking management system for smart campus, Internet of Things, Volume 14, 2021, pp. $100387 . \quad$ DOI: https://doi.org/10.1007/10722167_15

[15] Mukadam, Z. \& Logeswaran, R., A cloud-based smart parking system based on IoT technologies. Journal of Critical Reviews. 7 (3), 2020, p.pp. 105109 DOI: https://doi.org/ 10.31838/JCR.07.03.20

[16] Z. Guo, A. K. Bashir, K. Yu, J. C. Lin, Y. Shen, "Graph Embedding-based Intelligent Industrial Decision for Complex Sewage Treatment Processes", International Journal of Intelligent Systems, 2021, doi: 10.1002/int.22540.
[17] K. Yu, L. Tan, S. Mumtaz, S. Al-Rubaye, A. AlDulaimi, A. K. Bashir, F. A. Khan, "Securing Critical Infrastructures: Deep Learning-based Threat Detection in the IIoT", IEEE Communications Magazine, 2021.

[18] L. Zhen, A. K. Bashir, K. Yu, Y. D. Al-Otaibi, C. H. Foh, and P. Xiao, "Energy-Efficient Random Access for LEO Satellite-Assisted 6G Internet of Remote Things", IEEE Internet of Things Journal, doi: 10.1109/JIOT.2020.3030856.

[19] L. Zhen, Y. Zhang, K. Yu, N. Kumar, A. Barnawi and Y. Xie, "Early Collision Detection for Massive Random Access in Satellite-Based Internet of Things," IEEE Transactions on Vehicular Technology, vol. 70, no. 5, pp. 5184-5189, May 2021, doi: 10.1109/TVT.2021.3076015.

[20] Prabu, S., Velan, B., Jayasudha, F.V., Visu, P. and Janarthanan, K., 2020. Mobile technologies for contact tracing and prevention of COVID-19 positive cases: a cross-sectional study. International Journal of Pervasive Computing and Communications.

[21] Bhuvaneswary, N., Prabu, S., Karthikeyan, S., Kathirvel, R. and Saraswathi, T., 2021. Low Power Reversible Parallel and Serial Binary Adder/Subtractor. Further Advances in Internet of Things in Biomedical and Cyber Physical Systems, p.151.

[22] Nguyen, N.T., Leu, M.C., Zeadally, S., Liu, B.H. and Chu, S.I., 2018. Optimal solution for data collision avoidance in radio frequency identification networks. Internet Technology Letters, 1(3), p.e49.

[23] Do, D.T., Le, T.A., Nguyen, T.N., Li, X. and Rabie, K.M., 2020. Joint impacts of imperfect CSI and imperfect SIC in cognitive radio-assisted NOMAV2X communications. IEEE Access, 8, pp.128629128645 .

[24] Hiremath, P.N., Armentrout, J., Vu, S., Nguyen, T.N., Minh, Q.T. and Phung, P.H., 2019, November. MyWebGuard: toward a user-oriented tool for security and privacy protection on the web. In International Conference on Future Data and Security Engineering (pp. 506-525). Springer, Cham.

[25] Shivappriya, S.N., Karthikeyan, S., Prabu, S., Pérez de Prado, R. and Parameshachari, B.D., 2020. A modified ABC-SQP-based combined approach for the optimization of a parallel hybrid electric vehicle. Energies, 13(17), p.4529.

[26] Shivappriya, S.N., Priyadarsini, M., Stateczny, A., Puttamadappa, C. and Parameshachari, B.D., 2021. 
Cascade object detection and remote sensing object detection method based on trainable activation function. Remote Sensing, 13(2), p.200.

[27] Kowsalya, T., Babu, R.G., Parameshachari, B.D., Nayyar, A. and Mehmood, R.M., 2021. Low Area PRESENT Cryptography in FPGA Using TRNGPRNG Key Generation. CMC-COMPUTERS MATERIALS \& CONTINUA, 68(2), pp.14471465.

[28] Kiran, P., Parameshachari, B.D., Yashwanth, J. and Bharath, K.N., 2021. Offline Signature Recognition Using Image Processing Techniques and Back Propagation Neuron Network System. SN Computer Science, 2(3), pp.1-8. 